\title{
Is hyperglycemia seen in children during cardiopulmonary bypass a result of hyperoxia?
}

Karim S. Bandali, BSc, MSc

Michael P. Belanger, AALAS

Carin Wittnich, MSc, DVM

From the Departments of Physiology and Surgery, The Hospital for Sick Children, University of Toronto, Toronto, Ontario, Canada.

Supported by The Heart \& Stroke Foundation of Ontario (grant T2454). Karim $\mathrm{S}$. Bandali is supported by a fellowship from The Natural Sciences and Engineering Research Council of Canada (NSERC).

Received for publication Nov 30, 2000; revisions requested Jan 22, 2001; revisions received March 3, 2001; accepted for publication March 9, 2001.

Address for reprints: C. Wittnich, MSc, DVM, University of Toronto, Medical Sciences Building, Room 7256, 1 King's College Circle, Toronto, Ontario M5S 1A8, Canada (E-mail: c.wittnich@utoronto.ca).

J Thorac Cardiovasc Surg 2001;122:753-8

Copyright (C) 2001 by The American Association for Thoracic Surgery

0022-5223/2001 $\$ 35.00+0 \quad \mathbf{1 2 / 1 / 1 1 5 7 0 2}$

doi:10.1067/mtc.2001.115702
Objective: We sought to identify whether elevated $\mathrm{PaO}_{2}$ itself can directly cause hyperglycemia in newborns and to document any additional effects of cardiopulmonary bypass on this response.

Methods: Piglets were exposed to either normoxia $(88 \pm 6 \mathrm{~mm} \mathrm{Hg})$ or hyperoxia $(470 \pm 28 \mathrm{~mm} \mathrm{Hg})$ in the following studies. Anesthetized 3-day-old neonatal pigs were either ventilated for 2 hours of normoxia $(n=5)$ or hyperoxia $(n=5)$ or placed on normothermic, normoxic cardiopulmonary bypass $(\mathrm{n}=6)$ and then randomly assigned to either undergo a 2-hour normoxic period or a 1-hour hyperoxic episode, followed by a return to normoxia for an additional hour. Blood glucose levels were measured in all animals.

Results: No significant changes were observed in blood glucose levels in neonatal pigs that underwent 2 hours of normoxic ventilation $(5.0 \pm 0.6 \mathrm{mmol} / \mathrm{L})$ or cardiopulmonary bypass $(6.6 \pm 1.6 \mathrm{mmol} / \mathrm{L})$. However, the ventilatory model showed a significant and sustained $(P<.001)$ hyperglycemic response after both 1 hour $(8.6$ $\pm 1.0 \mathrm{mmol} / \mathrm{L})$ and 2 hours $(9.8 \pm 1.6 \mathrm{mmol} / \mathrm{L})$ of hyperoxia. In the cardiopulmonary bypass model, exposure to 1 hour of hyperoxia elicited a significant $(P<$ $.05)$ hyperglycemic response $(10.3 \pm 1.2 \mathrm{mmol} / \mathrm{L})$, followed by a return to normal blood glucose levels $(6.6 \pm 1.6 \mathrm{mmol} / \mathrm{L})$ with a return to normoxia. This hyperoxia-mediated hyperglycemic response was confirmed when data examined from children undergoing cardiopulmonary bypass for primary repair of their congenital defects also identified a significant positive correlation $(r=0.72, P=.02)$ between oxygen levels and blood glucose levels measured before and at the end of cardiopulmonary bypass.

Conclusions: Hyperoxia triggers a hyperglycemic response in both ventilatory and bypass models. Cardiopulmonary bypass does not exacerbate this response, as shown by the similar levels of hyperglycemia sustained for the duration of the hyperoxic exposure in both experimental models. Therefore, not only may hyperoxia play a crucial role in the hyperglycemic response seen during neonatal cardiopulmonary bypass, but its effect on glucose homeostasis should be considered whenever children are exposed to hyperoxia.

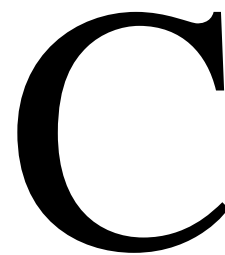

hildren undergoing surgical repair for congenital heart disease are often placed on cardiopulmonary bypass (CPB) during cardiac operations. ${ }^{1-3}$ The use of deep hypothermia in combination with $\mathrm{CPB}$ was thought to provide optimal conditions for cardiac surgical repair ${ }^{4-7}$ but can result in hyperglycemia. ${ }^{8}$ Persistent hyperglycemia in children has been shown to cause osmotic diuresis, dehydration, glycosylation of proteins, and an increased incidence of cerebral hemorrhage. ${ }^{9,10}$ 


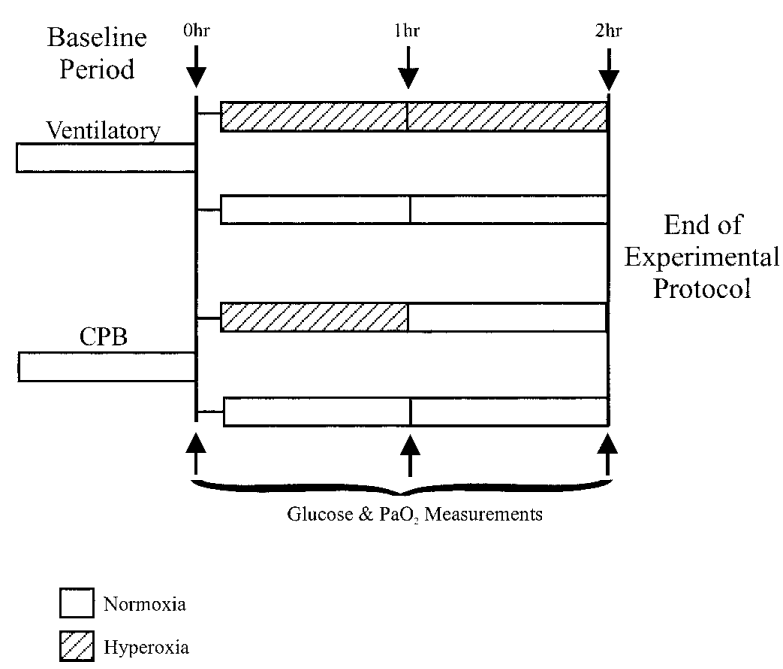

Figure 1. Surgical protocols for CPB and the ventilatory models. Hatched and open areas represent episodes of hyperoxia and normoxia, respectively.

Hypothermia-induced reductions in insulin secretion by inhibition of the pancreatic secretory process has been thought to be the cause of this hyperglycemic response that is commonly seen in children placed on CPB for cardiac repair. ${ }^{5,8,11-13}$ However, other evidence in the literature has indicated that hypothermia may not be the primary factor. ${ }^{8}$ In fact, hyperglycemic responses in adults undergoing cardiac repair have been documented, even under normothermic conditions. ${ }^{14}$ Whether the hyperglycemic response seen in children undergoing cardiac repair persists under normothermic conditions has not been addressed.

Other factors in $\mathrm{CPB}$, such as hypokalemia and hypocalcemia, have also been thought to contribute to the hyperglycemic response through the inhibition of insulin release. ${ }^{5,15,16}$ However, studies in adults have demonstrated that neither of these factors is consistently implicated. ${ }^{5,8,17-19}$ Other studies with adult patients have also speculated that hyperglycemia during CPB is caused by exogenous administration of glucose, either in intravenous or cardioplegic solutions, ${ }^{20,21}$ whereas others have pointed to the importance of enhanced reabsorption of filtered glucose in the development of hyperglycemia during CPB. ${ }^{22}$ Therefore, the primary factor responsible for the hyperglycemic response seen in patients undergoing cardiac operations remains controversial, and moreover, the primary factor that causes hyperglycemia in children remains unresolved.

In the majority of neonatal CPB cases, high oxygen levels (hyperoxia) have been used ${ }^{23}$; however, the effect of hyperoxia on neonatal glucose homeostasis remains essentially unexplored.

Although multivariate analysis may be used in patients to statistically determine the contribution of hyperoxia to
TABLE 1. Measurements of blood glucose and $\mathrm{PaO}_{2}$ at baseline and 2 hours of normoxia

\begin{tabular}{llcc}
\hline Protocol & \multicolumn{1}{c}{ Parameter } & $\begin{array}{c}\text { Baseline } \\
\text { value }\end{array}$ & $\begin{array}{c}\text { 2 Hours } \\
\text { of normoxia }\end{array}$ \\
\hline Ventilation & Glucose $(\mathrm{mmol} / \mathrm{L})$ & $5.0 \pm 1.0$ & $5.3 \pm 0.3$ \\
& $\mathrm{PaO}_{2}(\mathrm{~mm} \mathrm{Hg})$ & $86 \pm 9$ & $84 \pm 7$ \\
$\mathrm{CPB}$ & $\mathrm{Glucose}(\mathrm{mmol} / \mathrm{L})$ & $5.6 \pm 1.6$ & $6.6 \pm 1.6$ \\
& $\mathrm{PaO}_{2}(\mathrm{~mm} \mathrm{Hg})$ & $84 \pm 20$ & $96 \pm 14$ \\
\hline
\end{tabular}

$C P B$, Cardiopulmonary bypass.

hyperglycemia, the physiologic effect of hyperoxia on glucose metabolism in the presence of confounding variables cannot be ignored. For example, the presence of heart disease or varying degrees of cyanosis may itself affect glucose handling and the hyperglycemic response. The models used in this study provide the unique opportunity to examine the pure effect of oxygen on neonatal blood glucose levels in a controlled experimental setting with a ventilatory model in the absence of CPB because CPB is also associated with the release of several neurohumoral factors. ${ }^{24,25}$ Whether these factors can contribute to the hyperglycemic response is unknown. Therefore, a CPB model was used to determine whether CPB exaggerates the potential hyperoxia-mediated hyperglycemia and whether this response was rapidly reversible with a return to normoxia.

\section{Methods}

Neonatal Yorkshire pigs were chosen as the animal model in which to study the effects of hyperoxia because the cardiac and pulmonary systems of newborn human subjects and pigs have many structural and functional similarities. ${ }^{26,27}$ Yorkshire pigs (3 days old, 1.5-2.5 $\mathrm{kg}$ ) were anesthetized with an intraperitoneal injection of sodium pentobarbital (65 mg/kg; MTC Pharmaceuticals, Cambridge, Ontario, Canada), intubated, and mechanically ventilated to normal blood gases $\left(\mathrm{PaO}_{2}, 88 \pm 6 \mathrm{~mm} \mathrm{Hg} ; \mathrm{PaCO}_{2}, 38 \pm 5 \mathrm{~mm} \mathrm{Hg}\right)$ with medical air. Normothermia $\left(37.9^{\circ} \mathrm{C} \pm 0.2^{\circ} \mathrm{C}\right)$ was maintained in each piglet. A catheter was inserted into the right carotid artery and advanced to the aortic arch to monitor arterial blood pressure through a pressure transducer (Cobe Laboratories, Lakewood, Colo), which was connected to a physiologic recorder (Biopac Systems Inc, Goleta, Calif). This catheter was also used for sampling of blood gases and glucose measurements.

Arterial blood gases $\left(\mathrm{PaO}_{2}\right.$ and $\left.\mathrm{PaCO}_{2}\right)$ and acid-base status $(\mathrm{pH}$ and bicarbonate $\left[\mathrm{HCO}^{3-}\right]$ ) were monitored at regular intervals with an ABL30 Acid-Base Analyzer (Radiometer, Copenhagen, Denmark), and appropriate ventilatory adjustments ensured that $\mathrm{PaO}_{2}$ and $\mathrm{PaCO}_{2}$ remained at the desired levels.

Blood glucose measurements were made with the Accu-Chek Advantage Blood Glucose Monitoring System (Boerhinger Mannheim, Laval, Quebec, Canada). Measurements of both $\mathrm{PaO}_{2}$ and blood glucose levels were made at 0-, 1-, and 2-hour intervals (Figure 1). Plasma insulin measurements were made with a porcine insulin enzyme-linked immunosorbent assay (ALPCO Diagnostics, Windham, $\mathrm{NH}$ ) from samples taken both at the 0 - and 

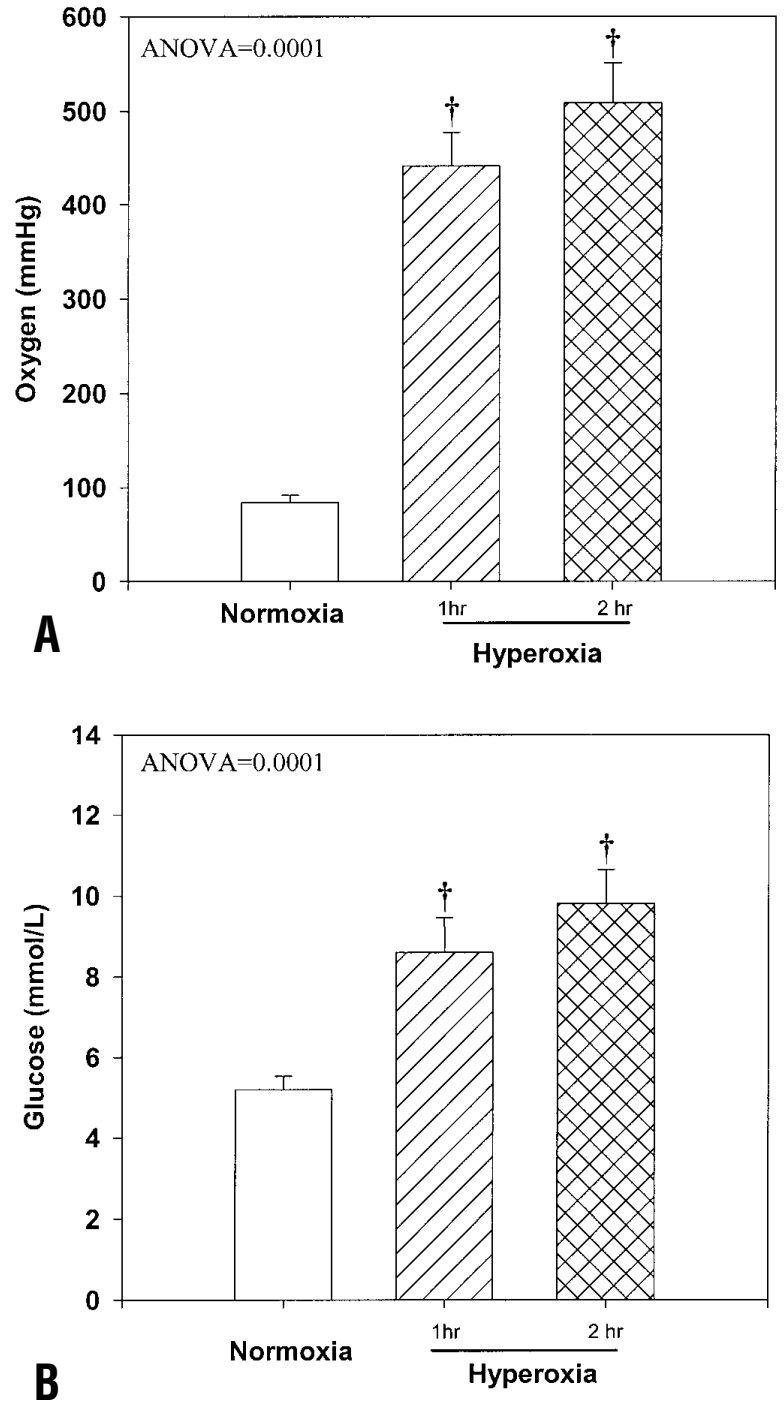

Figure 2. Neonatal oxygen levels (A) and blood glucose levels (B) during 2 hours of hyperoxic ventilation. Post hoc analysis yielded the following statistically significant differences: $t P<.001$ versus normoxia. ANOVA, Analysis of variance.

2-hour intervals from representative normoxic $(\mathrm{n}=4)$ and hyperoxic $(n=3)$ piglets in the ventilatory model.

In those piglets placed on normoxic, normothermic CPB with a Sarns 9000 perfusion system (3M/Sarns, Inc, Ann Arbor, Mich), systemic heparinization (heparin sulfate, $400 \mathrm{IU} / \mathrm{kg}$; Leo Pharma Inc, Ajax, Ontario, Canada) was instituted after sternotomy was performed. Appropriate perfusion adjustments maintained mean arterial blood pressure $(61 \pm 1 \mathrm{~mm} \mathrm{Hg})$, and blood gases were maintained with a Minimax membrane oxygenator (Medtronic, Inc, Anaheim, Calif). Heparin was administered to maintain an activated clotting time of greater than 400 seconds, sodium bicarbonate was administered to maintain $\mathrm{pH}(7.40 \pm 0.02)$, and sodium pentobarbital was administered to ensure a surgical plane of anesthesia.

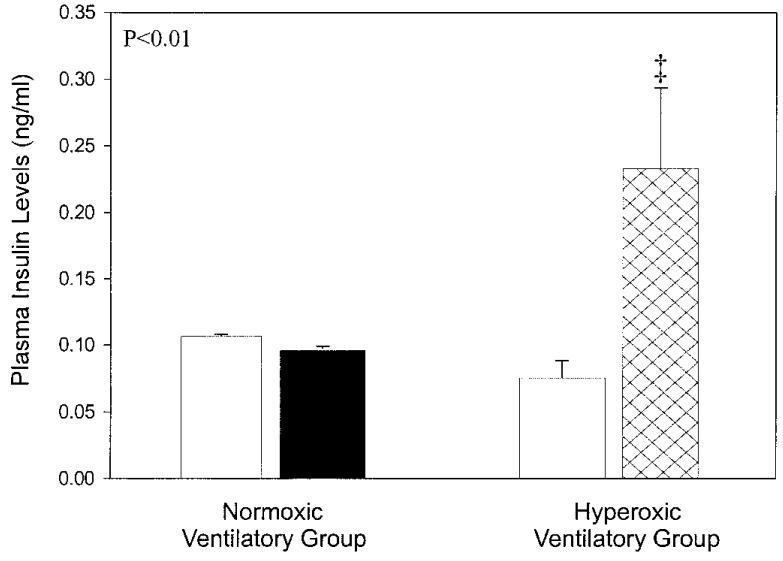

Figure 3. Plasma insulin levels measured in piglets at baseline (open bar) and at the end of either 2 hours of normoxic (filled bar) or hyperoxic (cross-hatched bar) ventilation. Paired $t$-test analysis yielded the following statistically significant differences: $¥ P<.01$ versus baseline.

\section{Experimental Protocol}

A 2-hour ventilatory model was developed in which animals were randomly allocated to normoxic control $(\mathrm{n}=5)$ or hyperoxic $(\mathrm{n}=$ 5; 1 hour: $441 \pm 36 \mathrm{~mm} \mathrm{Hg}, 2$ hours: $509 \pm 42 \mathrm{~mm} \mathrm{Hg}$ ) study groups (Figure 1). So that we could determine whether CPB exaggerated the potential hyperoxia-mediated hyperglycemia response, an additional 6 neonatal pigs were placed on normothermic, normoxic CPB. Three of these piglets underwent a 1-hour hyperoxic ( $304 \pm 145 \mathrm{~mm} \mathrm{Hg}$ ) episode and were then returned to normoxia for an additional hour, and the remaining 3 piglets underwent a 2hour exposure to normoxia (Figure 1).

All experimental procedures and protocols used in this investigation were reviewed and approved by the University of Toronto Animal Care and Use Committee and are in accordance with the "Guide for the Care and Use of Laboratory Animals" prepared by the Institute of Laboratory Animal Resources, National Research Council, and published by the National Academy Press, revised 1996, and Canadian Council on Animal Care guidelines.

\section{Statistical Analysis}

One-way analysis of variance with the Tukey post hoc test was used to analyze differences in blood glucose levels in the animals placed on CPB. Repeated-measures analysis of variance with the Duncan multiple-range post hoc test was used to identify differences between normoxic and hyperoxic groups across time in the ventilatory model. Differences in plasma insulin levels measured from ventilated animals were compared by means of paired $t$-test analysis. All data are expressed as means \pm standard deviation.

\section{Results}

\section{Ventilatory Model}

No significant changes were observed in blood glucose levels in neonatal pigs that underwent 2 hours of normoxic ventilation (Table 1). In contrast, after initial normoxia, ini- 

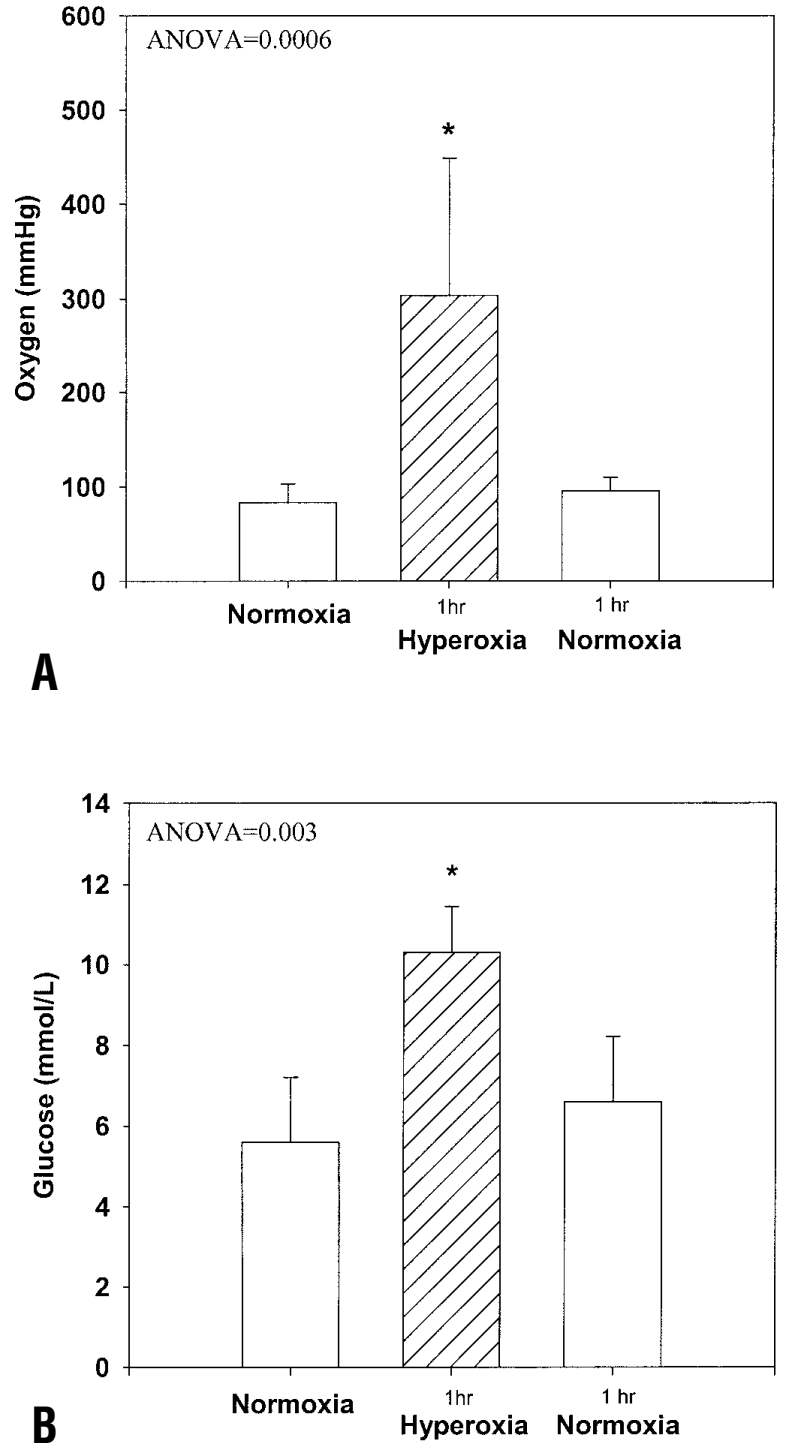

Figure 4. Neonatal oxygen levels (A) and blood glucose levels (B) during 2 hours of CPB. Post hoc analysis yielded the following statistically significant differences: ${ }^{*} P<.05$ versus normoxia. ANOVA, Analysis of variance.

tiation of hyperoxia was confirmed by increases in $\mathrm{PaO}_{2}(P$ $<.001$ ) throughout the 2-hour period (Figure 2, A). Blood glucose levels increased significantly by $40 \%$ during the first hour and were further increased by $12 \%$ in the second hour of hyperoxia (Figure 2, B). No significant changes were observed in plasma insulin levels in piglets that underwent 2 hours of normoxic ventilation (Figure 3). However, in contrast, plasma insulin levels were significantly increased $(P<.01)$ by 3 -fold at the end of the 2-hour hyperoxic ventilatory period (Figure 3 ).

\section{CPB Model}

No significant changes were observed in blood glucose levels in neonatal pigs that underwent 2 hours of normoxic $\mathrm{CPB}$ (Table 1). Figure 4, $A$, shows that with the induction of hyperoxia, $\mathrm{PaO}_{2}$ levels were appropriately significantly elevated during 1 hour $(P<.05)$ of hyperoxia and successfully returned to normoxia for another hour. Blood glucose levels (Figure $4, B$ ) of neonatal pigs that underwent normothermic hyperoxic $\mathrm{CPB}$ began within the normal physiologic range during the initial normoxic exposure. In response to 1 hour of hyperoxia, blood glucose levels showed a significant $46 \%$ increase $(P<.05)$. Once normoxia was re-established, blood glucose was then shown to quickly return to normal $(P<.05)$.

These data illustrated that hyperoxia-induced hyperglycemia occurred in both ventilatory and CPB normothermic models and returned to normal when normoxia was restored, thereby confirming the effect of hyperoxia alone on neonatal blood glucose levels. Similar levels of hyperglycemia sustained for the duration of the hyperoxic exposure in both experimental models also suggest that CPB does not exacerbate the hyperglycemic response.

\section{Discussion}

Hyperglycemia is frequently observed in children during CPB. Although the underlying cause of this response during CPB has been historically ascribed to a decrease in insulin secretion mediated by such factors as hypothermia, hypocalcemia, and hypokalemia, ${ }^{5,15,16}$ none of these factors has been consistently implicated. ${ }^{5-8,17-19}$ The inconsistency in the outcome of these studies may be partly explained by the fact that a majority of these studies do not evaluate hyperglycemia as an independent variable without the presence of confounding variables, such as congenital heart defects, cyanosis, and preoperative medications. Moreover, the potential contribution of hyperoxia commonly used in neonatal CPB to the hyperglycemic response remains essentially unexplored.

Because the newborn pig model closely resembles the structure, function, and cardiovascular physiology of a newborn child, ${ }^{26,27}$ the models used in this study provided an opportunity to identify the potential relationship between hyperglycemia and $\mathrm{PaO}_{2}$ in the absence of confounding variables normally found in pediatric patients. The ventilatory model was first used to clearly identify the pure effect of oxygen on neonatal blood glucose levels in the absence of CPB by using a 2-hour protocol of severe hyperoxia and showed a significant hyperoxia-induced hyperglycemic response. Once this relationship was established, a CPB model with clinically relevant levels of hyperoxia was used to determine whether CPB exaggerated the hyperoxia-mediated hyperglycemic response and whether this response was rapidly reversible in the presence of normoxia. This study clearly demonstrates that similar hyperglycemic levels 
resulted by using 2 different models and hyperoxic levels, therefore suggesting that $\mathrm{CPB}$ does not potentiate the hyperglycemic response. Furthermore, neonatal blood glucose levels quickly returned to normal in the CPB model once normoxia was re-established. Therefore, not only may hyperoxia play a crucial role in the hyperglycemic response seen in neonatal CPB, but its effect on glucose homeostasis should be considered whenever children are exposed to hyperoxia.

This was borne out when 5 children between 11 and 18 months of age who underwent routine CPB for primary repair of their congenital defects at the Hospital for Sick Children (Toronto, Canada) were studied. They had normal preoperative blood glucose levels, and during the operation, they demonstrated a significant and strong positive correlation $(r=0.72, P=.02)$ between their absolute oxygen and blood glucose levels (Figure 5), confirming that hyperoxia is indeed an important contributor to the hyperglycemic response seen in children who are placed on $\mathrm{CPB}$ during primary repair for congenital heart defects. It should be noted that the hyperoxic levels used in our CPB piglet model in this study were similar to the levels seen in this clinical work, and because of some variability in the actual degree of hyperoxia attained, the CPB model also had a strong positive correlation between oxygen and glucose levels $(r=0.84, P=.03$ ). The 2-hour time course chosen for both our experimental models was based on similar time courses commonly seen in children undergoing $\mathrm{CPB}$ for primary cardiac repair.

Although this work illustrates that hyperoxia is an important and major contributor to the hyperglycemia seen in neonatal $\mathrm{CPB}$, the potential contribution of hypothermia, electrolyte differences, inotropic agents, and exogenous glucose (depending on rate and concentration of administration) cannot be ruled out, despite never being consistently implicated in past studies. These and other factors, such as the presence of metabolic alkalosis and variable rates of glucose uptake, as seen in a recent study, ${ }^{28}$ may potentially exacerbate or blunt the hyperoxia-induced hyperglycemic response seen during neonatal CPB.

The mechanisms that underlie the hyperoxia-induced hyperglycemic response may involve alterations in enzymes or other glucose regulators. Studies examining the effects of extreme hyperbaric hyperoxia have shown inhibitory effects on many metabolic enzymes that are important in both glycolysis and aerobic metabolism. ${ }^{29}$ In addition, some studies have implicated reductions in insulin secretion as a potential contributor to the hyperglycemia seen in children placed on CPB for cardiac repair. ${ }^{5,8,11-13}$ However, in contrast to these studies, insulin levels measured in piglets exposed to hyperoxia for 2 hours showed a significant 3 -fold elevation. This may implicate insulin resistance as one possible mechanism for the hyperoxia-mediated hyperglycemic response. In

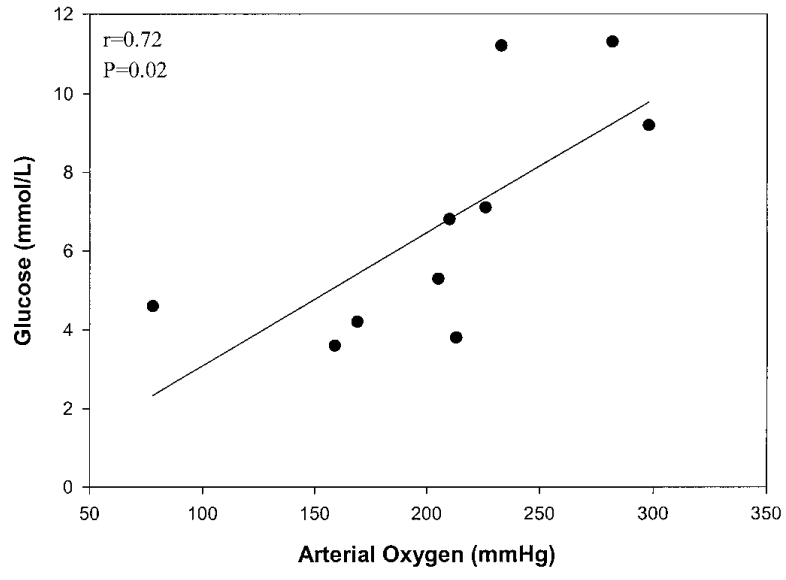

Figure 5. Significant correlation $(P=.02, r=0.72)$ between oxygen and blood glucose levels in children undergoing standard CPB in the neonatal period for primary repair of tetralogy of Fallot.

addition, it is interesting to note that the development of hyperglycemia in both the ventilatory and CPB model occurred within 15 minutes of the hyperoxia exposure. In light of this rapidly induced hyperglycemia, the potential role of hormonal changes, such as insulin levels, may be particularly important in elucidating the mechanisms that underlie this response.

The development of hyperglycemia in children has been shown to cause osmotic diuresis, dehydration, and the glycosylation of proteins. ${ }^{9}$ The most serious potential effect of hyperglycemia has been emphasized by studies that demonstrate increased mortality. ${ }^{9}, 10$ Specifically, a certain cohort of low-birth-weight newborn infants receiving parenteral glucose at birth had persistent hyperglycemia during the first 24 hours and were at high risk of having a cerebral hemorrhage. ${ }^{9,10}$ Thus, depending on the magnitude and duration of the hyperglycemic response to hyperoxia, these detrimental effects could also be of potential concern in children undergoing CPB.

In addition to the hyperglycemic response triggered by the use of hyperoxia during $\mathrm{CPB}$, hyperoxia itself may detrimentally affect neonates undergoing primary cardiac repair. The potential detrimental effects of hyperoxia have been alluded to in the literature. ${ }^{23,30}$ The original rationale for the use of hyperoxia during cardiac operations was to overcome the hypothermia-induced leftward shift of the oxygen-hemoglobin dissociation curve and thus ensure adequate oxygen delivery. Although some degree of hyperoxia may be essential to ensure adequate oxygenation under hypothermic conditions, the rationale for the use of hyperoxia under normothermic conditions is unclear. ${ }^{23}$ Furthermore, a recent study reports that although hyperoxia provides a myocardial energy-sparing effect during early 
ischemia, including minimized reductions in myocardial adenosine triphosphate and glycogen content, it also increased the incidence of ventricular fibrillation, which negated this beneficial metabolic effect. ${ }^{23}$

Work by Ihnken and colleagues ${ }^{30}$ has also shown that the use of hyperoxia in immature hypoxemic piglet hearts during CPB resulted in increased susceptibility to oxygenmediated damage after reintroduction of molecular oxygen. This unintended reoxygenation injury causes lipid peroxidation, enzyme release, endothelial and intracellular lesions leading to membrane damage, and reduced myocardial performance. Furthermore, the authors went on to show that reductions in high oxygen levels in the bypass prime and in blood cardioplegic solution to normoxia can reduce the damaging changes associated with hyperoxia and improve contractility in previously hypoxic pig hearts.

The current study shows that hyperoxia itself can elicit a hyperglycemic response as seen in neonatal pigs in both a ventilatory and CPB model. This hyperglycemic response is abolished with the reduction of oxygen levels to normoxia. Moreover, clinical data in children undergoing cardiac operations show a strong positive correlation between $\mathrm{PaO}_{2}$ and glucose levels, confirming the animal findings. The relationship between $\mathrm{PaO}_{2}$ and glucose elucidated in these children appear dose-dependent until severe hyperoxic levels are reached. Therefore, in addition to the increased incidence of ventricular fibrillation and oxygen-mediated damage that has been shown in animal studies to accompany unintended reoxygenation injury, the use of hyperoxia in children during CPB does appear to contribute to alterations in blood glucose homeostasis. This may potentially contribute to postoperative complications seen in certain children undergoing cardiac operations. Therefore, for neonatal myocardial protection to be optimized, consideration may need to be given to adjustments in the optimal levels of oxygen used.

We acknowledge the contributions of Cathy Carlyle, Shona M. Torrance, and W. Jack Wallen.

\section{References}

1. Barratt-Boyes BG, Neutze JM, Seelye ER, Simpson M. Complete correction of cardiovascular malformations in the first year of life. Prog Cardiovasc Dis. 1972;15:229-53.

2. Pooley RW, Hayes CJ, Edie RN, Gersony WM, Bowman FO, Malm JR. Open-heart experience in infants using normothermia and deep hypothermia. Ann Thorac Surg. 1976;22:415-23.

3. Wittnich C. Pediatric cardiopulmonary bypass and heart preservation. Can J Pediatr. 1991;3:6-13.

4. Mori M, Muraoka R, Yokoto Y, Okamoto Y, Ando F, Fukumasu H, et al. Deep hypothermia combined with cardiopulmonary bypass for cardiac surgery in neonates and infants. $J$ Thorac Cardiovasc Surg. 1972;64:422-9.

5. Brown TCK, Dunlop ME, Stevens BJ, Clarke CP, Shanahan FA. Biochemical changes during surface cooling for deep hypothermia in open-heart surgery. J Thorac Cardiovasc Surg. 1972;65:402-8.
6. Black PR, Van Devanter S, Cohn LH. Current research review: effects of hypothermia on systemic and organ system metabolism and function. J Surg Res. 1976;20:49-63.

7. Brans YW, Dweck HS, Harris HB, Parr GVS, Bailey PE, Kirklin JW. Effect of open-heart surgery on the body composition of infants and young children. Pediatr Res. 1981;15:1024-8.

8. Benzing G, Francis PD, Kaplan S, Helmsworth JA, Sperling MA Glucose and insulin changes in infants and children undergoing hypothermic open-heart surgery. Am J Cardiol. 1983;52:133-6.

9. Plides RS. Neonatal hyperglycemia. J Pediatr. 1986;109:905-7.

10. Dweck HS, Cassady G. Glucose intolerance in infants of very low birth weights: incidence of hyperglycemia in birth weights $1100 \mathrm{gms}$ or less. Pediatrics. 1974;53:189-95.

11. Baum D, Dillard DH, Porte D Jr. Inhibition of insulin release in infants undergoing deep hypothermia cardiovascular surgery. $N$ Engl J Med. 1968;279:1309-18.

12. Johnson AE, Radde IC, Steward DJ, Taylor J. Acid-base and electrolyte changes in infants undergoing profound hypothermia for surgical corrections of congenital heart disease. Can Anaesth Soc J. 1974;21:23-45.

13. Landymore RW, Murphy DA, Longely WJ. Effect of cardiopulmonary bypass and hypothermia on pancreatic endocrine function and peripheral utilization of glucose. Can J Surg. 1979;22:248-50.

14. Kuntschen FR, Galletti PM, Hahn C. Glucose-insulin interactions during cadiopulmonary bypass: hypothermia versus normothermia. $J$ Thorac Cardiovasc Surg. 1986;91:451-9.

15. Howell SL, Taylor KW. Potassium ions and secretions of insulin by Islets of Langerhans incubated in vitro. Biochem J. 1968;108:17-24.

16. Curry DL, Curry KP. Hypothermia and insulin secretion. Endocrinology. 1970;87:750-5.

17. Mandelbaum I, Morgan CR. Effect of extracorporeal circulation upon insulin. J Thorac Cardiovasc Surg. 1968;55:526-34.

18. Levine R. Mechanisms of insulin secretion. $N$ Engl $J$ Med. 1970;272:522-6

19. Moffitt EA, Roseavear JW, Molnar GD, McGoon DC. Myocardial metabolism in open-heart surgery: correlation with insulin response. $J$ Thorac Cardiovasc Surg. 1970;59:691-706.

20. Kuntschen FR, Galetti PM, Hahn C, Amulf JJ, Isetta C, Dor V. Alterations of insulin and glucose metabolism during cardiopulmonary bypass under normothermia. $J$ Thorac Cardiovasc Surg. 1985;89:97-106.

21. Mescheryakov AV, Kozlov IA, Dementyeva II, Lakhter MA, Ermolyn AF, Galyibin AA. Glucose metabolism and insulin activity during cardiac surgery. J Cardiothorac Anesth. 1989;3:536-43.

22. Braden H, Cheema-Dhadli S, Mazer CD, McKnight DJ, Singer W, Halperin ML. Hyperglycemia during normothermic cardiopulmonary bypass: the role of the kidney. Ann Thorac Surg. 1998;65:1588-93.

23. Wittnich C, Torrance SM, Carlyle CE. Effects of clinical levels of hyperoxia on neonatal myocardial energy status and responses to global ischemia. Ann Thorac Surg. 2000;70:2125-31.

24. Saatvedt K, Lindberg H, Geiran OR, Michelsen S, Pedersen T, Seem E, et al. Release of interleuken- 8 and calprotectin and after paediatric cardiopulmonary bypass with and without ultrafiltration. Scand $J$ Thorac Cardiovasc Surg. 1996;30:53-9.

25. Withington DE, Aranda JV. Histamine release during cardiopulmonary bypass in neonates and infants. Can J Anesth. 1997;44:610-6.

26. DeRoth L, Downie HG. Basic cardiovascular parameters in the underweight neonatal swine. Biol Neonate. 1978;34:155-60.

27. Glauser EM. Advantages of piglets as experimental animals in pediatric research. Exp Med Surg. 1966;24:181-90.

28. Nollert G, Nagashima M, Bucerius J, Shin'oka T, Jonas R. Oxygenation strategy and neurological damage after deep hypothermic circulatory arrest. II. Hypoxic versus free radical injury. $J$ Thorac Cardiovasc Surg. 1999;117:1172-9.

29. Balentine JD, editor. Pathology of oxygen toxicity. New York: Academic Press; 1982

30. Ihnken K, Morita K, Buckberg GD, Young HH. Studies of hypoxemic/reoxygenation injury with aortic clamping. XI. Cardiac advantages of normoxemic versus hyperoxic management during cardiopulmonary bypass. J Thorac Cardiovasc Surg. 1995;110:1255-64. 